\title{
Etude De La Qualité Agronomique Et Biochimique De Quelques Variétés De Niébé (Vigna Unguiculata (L) Walp (Fabaceae) Provenant De La Côte d'Ivoire
}

\author{
Boyé Mambe Auguste Denise \\ Yapo Sopie Edwige Salomé \\ Koffi N'dodo Boni Clovis \\ Kouassi N'dri Jacob \\ Tonessia Dolou Charlotte \\ Soko Dago Faustin \\ Ballo Esperence Kouadio \\ Seu Jonathan Gogbeu \\ Ayolié Koutoua \\ Kouadio Yatty Justin \\ Université Jean Lorougnon Guédé,
}

Laboratoire de physiologie et pathologie végétale, Daloa, Côte d’Ivoire

doi: 10.19044/esj.2016.v12n24p362 URL:http://dx.doi.org/10.19044/esj.2016.v12n24p362

\begin{abstract}
Cowpea is a legume consumed in Côte d'Ivoire. There are many cultivars whose seeds are widely available. This study aimed to determine some agronomic and physicochemical characteristics of some cowpea cultivars in order to identify differences and if possible select the best for appropriate uses. The study included 16 cultivars; the agro-morphological variability was assessed on the basis of eight parameters. The results showed that the accessions N21DR, N18ZR, N10BBrp produced more seeds in a relatively short growing cycle. For the biochemical characterization, parameters such as moisture, ash, protein, fat, total carbohydrates and energy value were determined by conventional methods. Differences were observed between the physicochemical parameters of the analyzed cultivars seeds. The cultivar (N19ZBoBp) recorded the highest protein content.
\end{abstract}

Keywords: Cowpea, chemical composition, agronomic parameters, food safety, productivity 


\section{Résumé}

Le niébé est une légumineuse consommée en Côte d’Ivoire. Il existe de nombreux cultivars dont les graines sont largement disponibles. La présente étude a eu pour objectif de déterminer quelques caractéristiques agronomiques et physicochimiques de quelques cultivars de niébé aux fins d’identifier des différences et sélectionner si possible les meilleurs pour des usages appropriés. L’étude a porté sur 16 accessions, la variabilité agromorphologique a été évaluée sur la base de huit paramètres. Les résultats ont montré que les accessions N21DR, N18ZR, N10BBrp ont produit plus de graines dans un cycle de culture relativement court. Pour la caractérisation biochimique, les paramètres tels que l'humidité, la teneur en cendres, les protéines, les lipides, les glucides totaux et la valeur énergétique ont été déterminés selon des méthodes conventionnelles. Des différences ont été observées entre les paramètres physicochimiques des graines de cultivars analysés. Le cultivar (N19ZBoBp) a enregistré la teneur la plus élevée en protéine.

Mots-clefs: Niébé, composition chimique, paramètres agronomiques, sécurité alimentaire, productivité

\section{Introduction}

Le niébé (Vigna unguiculata (L.) Walp) est la légumineuse la plus importante des régions tropicales d’Afrique (Jakai et Adalla, 1997). Sa culture joue un rôle très important dans l'équilibre nutritionnel et dans l'économie des populations rurales. Il se caractérise par sa richesse en protéine ; sur le plan alimentaire, il occupe une place de choix du fait qu'il constitue une importante source de protéines et d'énergie pour les hommes et les animaux dans les pays en développement où l'accès aux protéines d’origines animales est difficile (Bressani, 1997). La collecte et la caractérisation des écotypes et cultivars locaux détenus par les paysans sont des activités indispensables dans la stratégie de conservation de la biodiversité agricole du fait des pertes subies par les banques existantes dans le pays. Les pertes sont essentiellement dues aux mauvaises conditions de conservation. De plus, les changements climatiques notamment le raccourcissement des périodes pluviales ont entraîné l'abandon de plusieurs variétés locales à long cycle. Les abandons ont été suivis de pertes de cultivars créant donc une érosion génétique pour cette culture. En effet, dans certains pays, le niébé fournit plus de la moitié des protéines consommées et joue un rôle clé dans l'alimentation (Pasquet et al., 1997). En Afrique, le niébé est cultivé avant tout pour ses graines sèches, cuisinées sous les formes les plus diverses. Dans de nombreuses régions, on consomme aussi ses jeunes feuilles, fraîches ou séchées, et ses gousses immatures (Pasquet et al., 
1997; Balla, 2006). Selon Coulibaly et Lowenberg-Deboer (2002), l'Afrique Occidentale est actuellement loin de couvrir ses besoins en niébé par sa propre production. Une augmentation de la production pourrait donc générer d'importantes devises pour tous les acteurs de la filière.

En Côte d'Ivoire, bien que très consommé, le niébé demeure une culture marginale (.N'gbesso, 2013). La production avoisine les 36,310 tonnes/an, ce qui représente moins de $2 \%$ de la production africaine. Pourtant, le niébé, du fait de sa teneur relativement élevée en protéines devrait être valorisé dans plusieurs domaines. Il y a notamment, le domaine de farines composées et celui de l'enrichissement des aliments traditionnels. $\mathrm{Vu}$ son importance, très peu de données existent sur la composition agronomique et biochimique du niébé en Côte d'Ivoire, il est essentiel de caractériser certains cultivars locaux pour évaluer leur potentiel de production et leur composition biochimique. De plus, l'enrichissement des aliments traditionnels (à base de céréales) par adjonction de protéines végétales (légumineuses) étant susceptible d'améliorer leur valeur nutritionnelle (Afoakwa, 2004; Khalid, 2012). L’utilisation du niébé dans ce domaine pourrait donc contribuer à réduire les nombreux déficits ou carences alimentaires observés. L'objectif du présent travail est d'étudier les paramètres agronomiques et biochimiques de quelques variétés locales de niébé aux fins d'identifier les meilleures pour une éventuelle production à grande échelle et un enrichissement des aliments traditionnels.

\section{Matériel et méthodes}

Site d'étude

L'étude a été réalisée sur le site expérimental de l'Université Jean Lorougnon Guédé, située dans le département de Daloa. La ville est localisée dans la région du Haut Sassandra au Centre-Ouest de la Côte d'Ivoire entre le $6^{\circ}$ et $7^{\circ}$ de latitude Nord et le $7^{\circ}$ et $8^{\circ}$ de Longitude Ouest. Le substrat pédologique de Daloa appartient au vieux socle précambrien composé de granites, migmatites. Ces sols, lessivés et profonds $(20 \mathrm{~m})$ sont dus aux précipitations abondantes et à l'altération rapide des roches. Les sols de la région sont majoritairement ferralitiques (typiques). Ils sont généralement très profonds avec un taux élevé de matière organique. La pluviométrie, la température et l'humidité atmosphérique moyenne caractérisant le site d'étude dans les périodes d'essai de juillet à octobre (2014 et 2015) correspondant à la grande saison des pluies sont respectivement: 342,14 $\mathrm{mm} ; 25,97^{\circ} \mathrm{C}$ et $84,02 \%$. (Source : www.tutiempo.net).

\section{Dispositif experimental}

Le matériel végétal utilisé dans cette étude était constitué des graines de seize accessions de niébé. Les différents codes attribués à ces accessions 
(échantillons) ont été faits sur la base du numéro de l'accession, la provenance des graines, la couleur de l'enveloppe tégumentaire et la forme de la graine. Il s'agit des accessions suivantes : $\mathrm{N}_{2} \mathrm{KBoBg}, \mathrm{N}_{3} \mathrm{KR}$, N4KBNp, $\mathrm{N}_{5} \mathrm{BBr}, \mathrm{N}_{6} \mathrm{BR}, \mathrm{N}_{7} \mathrm{BRc}, \mathrm{N}_{8} \mathrm{BRcp}, \mathrm{N}_{9} \mathrm{BN}, \mathrm{N}_{10} \mathrm{BBrp}, \mathrm{N}_{11} \mathrm{BBoBp}, \mathrm{N}_{13} \mathrm{KBoNm}$, $\mathrm{N}_{14} \mathrm{BBoBg}, \mathrm{N}_{15} \mathrm{ZBoNg}, \mathrm{N}_{18} \mathrm{ZR}, \mathrm{N}_{19} Z B \mathrm{BoBp}$ et $\mathrm{N}_{21} \mathrm{DR}$. Les essais ont été réalisés sur une superficie de 0,25 ha $(50 \mathrm{~m}$ x $50 \mathrm{~m})$. Le dispositif expérimental est en blocs aléatoires complets avec trois répétitions. Chaque bloc est représenté par seize parcelles élémentaires comportant les seize accessions. Ainsi, sur l'ensemble des trois blocs, 48 parcelles élémentaires ( $3 \mathrm{~m} \times 1,5 \mathrm{~m}$ ) ont été mises en place. Les trois blocs sont séparés de $2 \mathrm{~m}$ les uns des autres et les parcelles à l'intérieur d'un bloc, de 0,5 m. Les semis ont été effectués pendant la grande saison des pluies correspondant à la période allant de mars à juillet. Une densité de 81818 plantes /ha soit 8,18 plantes $/ \mathrm{m}^{2}$ a été utilisée. Les semis ont été réalisés à raison de trois graines par poquet, à une profondeur de $3 \mathrm{~cm}$, avec un espacement de $30 \mathrm{~cm}$ x $30 \mathrm{~cm}$ pour la densité de semis. Chacune des parcelles élémentaires de $4,5 \mathrm{~m}^{2}$ a reçu 18 points de semis. Dix jours après semis (10 JAS), le démariage a été réalisé afin de ne conserver que la meilleure plante par poquet de semis. Après semis pour éviter un envahissement des plantes d'intérêts. Un traitement insecticide au lamdor 25 EC permettant de détruire les insectes a été effectué, 15 jours après semis.

\section{Collecte de données}

Toutes les mesures ont été effectuées sur cinq pieds pris au hasard par variété ou planche de façon manuelle. A la fin du cycle de la plante, les gousses sont récoltées par bloc et par parcelle élémentaire. Après la récolte, le nombre de gousses matures par plante a été dénombré. Le poids sec des gousses par plante a été déterminé après séchage au soleil jusqu'à l’obtention d'un poids constant. Les gousses ont ensuite été décortiquées pour compter le nombre de graines par plante. Le poids des graines et de 100 graines par plante ont été déterminés. Le poids des gousses vides a été évalué. L’indice de récolte ainsi que le taux de remplissage ont été déterminés. Les paramètres mesurés et les méthodes de mesures sont consignés dans le Tableau 1. En ce qui concerne les analyses biochimiques, les paramètres physicochimiques notamment les teneurs en eau, en protéines, en cendres, en glucides, en matières grasses et la valeur énergétique ont été déterminés. L'humidité, la teneur en protéines, la teneur en matières grasses et la teneur en cendres ont été évaluées selon la méthode BIPEA, (1976). La teneur en glucides a été déterminée par calcul selon l'expression suivante :

Glucidestotaux $=100$-[cendres(\%) +protéines(\%)+lipides (\%)] 
La valeur énergétique a été calculée en tenant compte des coefficients d'Atwater (FAO 2013) selon l'expression suivante: Valeur Energétique $(\mathrm{kcal})=[(\%$ glucides x 4$)+(\%$ protéines x 4$)+(\% \mathrm{MGx} 9)]$

\section{Analyse statistique des données}

Pour chacune des variables agronomiques et biochimiques, les moyennes ont été comparées en prenant en compte l'accession à travers une analyse de la variance à un facteur (ANOVA 1 ). La signification du test a été déterminée en comparant la probabilité $(P)$ associée à la statistique du test au seuil $\alpha=0,05$. Lorsqu'une différence significative a été observée entre les accessions, l'ANOVA a été complétée par le test de la Plus Petite Différence Significative (PPDS). La PPDS a permis d'identifier le ou les accessions qui induisent significativement cette différence.

\section{Resultats et discusions Résultats}

On remarque quel que soit la répétition, la tendance générale pour la majorité des variables analysées ne change pas. En conséquence, toutes les valeurs ont été combinées et seules les moyennes sont présentées. Les accessions N18ZR, N21DR, N11BBoBp et N14BBoBg n'ont pas fait l'objet d'analyse biochimique car ils ont été mélangés pendant le transport, donc ne pouvant pas les identifier, ces analyses n’ont pas été effectuées. Par contre, les accessions $\mathrm{N} 12 \mathrm{KBoBm}$ et $\mathrm{N} 17 \mathrm{ZBoNp}$ n’ont pas été évalués agro morphologiquement car ils ont perdu leur pouvoir germinatif.

\section{Caractérisation agromorphologique des accessions de niébé}

L'analyse de la variance a montré une différence hautement significative $(p<0,01)$ entre accessions pour les huit variables étudiés. Le nombre de gousses et le poids moyen des coques les plus élevés ont été obtenus avec l'accession N21DR avec respectivement 31 et 15,21g. Les accessions ayant les poids de 100 graines les plus élevés sont N2BoBg, N11BBoBp et N14BBoBg par contre, les accessions N3KR, N5BBr, N6BR, N7BRc, N8BRcp ont présenté les poids de 100 graines les plus faibles. Les accessions N8BRcp, N10BBrp, N18ZR, N21DR, N2BoBg ont produit les poids de graines les plus élevés avec respectivement 19,79 \pm 7,80, 20,15 \pm 5,54, 20,20 $\pm 5,69,1,49 \pm 3,19,19,59 \pm 1,96$. Les accessions N6BR, N8BRcp, N9BN, N10BBrp, N18ZR ont présenté des indices de récolte statiquement égaux. Par contre, l’indice de récolte le plus élevé a été obtenu avec l'accession N4KBNp avec une valeur de 0,5. Quant au taux de remplissage (TxR) le plus élevé, il a été obtenu avec l'accession N18ZR. 


\section{Caractérisation physico-chimiques des accessions de niébé}

Le tableau 3 présente les compositions chimiques moyennes des graines de chaque cultivar de niébé. L'humidité des graines a varié de $6,31 \pm 0,02 \%$ pour le cultivar N4KBNp à 9,33 $\pm 0,01 \%$ (cultivar $\mathrm{N} 12 \mathrm{KBoBm})$. Au niveau des cendres, les teneurs enregistrées ont varié de $3,83 \pm 0,15 \%$ (cultivar N15ZBoNg) à 4,90 $\pm 0,05 \%$ (cultivar N12KBoBm). De même, la teneur en matières grasses des graines a varié de 1,95 $\pm 0,08 \%$ (cultivar N1) à 4,01 $\pm 0,16 \%$ pour le cultivar N2KBoBg. Concernant la teneur en glucides, elle a varié de $61,44 \pm 0,14 \%$ (cultivar $\mathrm{N} 12 \mathrm{KBoBm}$ ) à $65,72 \pm 0,02 \%$ pour le cultivar N4KBNp. La teneur en protéines et la valeur énergétique de graines ont varié respectivement de 20,63 $\pm 0,02 \%$ (cultivar N3KR) à 23,31 $\pm 0,03 \%$ (cultivar N19ZBoBp) et de 362,25 $\pm 0,86 \mathrm{kcal} / 100 \mathrm{~g}$ (cultivar N13KBoNm) à 375,84 $\pm 0,78 \mathrm{kcal} / 100 \mathrm{~g}$ (cultivar N4KBNp). L'analyse de variance a montré une différence significative $(p<0,05)$ entre les accessions pour tous les paramètres. Les graines du cultivar N19ZBoBp ont présenté la teneur en protéines la plus élevée $(23,31 \pm 0,03 \%)$ alors que le

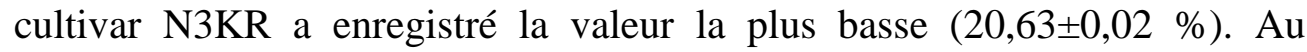
niveau de la teneur en matières grasses, les cultivars N2KBoBg, N15ZBoNg et $\mathrm{N} 5 \mathrm{BBr}$ ont enregistré les taux les plus élevées avec respectivement $4,01 \pm 0,16 \%, 3,79 \pm 0,04 \%$ et $3,65 \pm 0,06 \%$. Pendant que le cultivar N19ZBoBp a donné les teneurs en cendres $(4,18 \pm 0,05)$ la plus élevée, le cultivar N4KBNp a enregistré la valeur énergétique la plus élevée $(375,84 \pm 0,78 \mathrm{kcal} / 100 \mathrm{~g})$. En outre, le cultivar N17ZBoNp a donné les

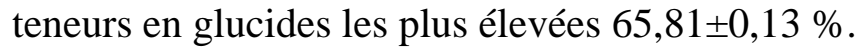

\section{Discussion}

\section{Caractérisation morphologique}

L'utilisation rationnelle de la diversité du niébé en Côte d'Ivoire exige une bonne connaissance des caractéristiques des accessions. La présente étude a permis d'identifier les accessions performantes. A la récolte, le nombre de gousses par plante a présenté des changements selon l'accession. L'accession N21DR a enregistré le nombre de gousses par plante le plus élevé avec 31 gousses en moyenne. Ce nombre a été inférieur à celui obtenu par Gbaguidi et al., (2015), ceux-ci ont obtenu 71 gousses en moyenne au cours d'une étude expérimentale qui a été conduite dans une zone soudano-guinéenne caractérisée par deux saisons pluvieuses et deux saisons sèches avec une pluviométrie moyenne de $1200 \mathrm{~mm} / \mathrm{an}$. Cette différence pourrait s'expliquer par le fait que nos conditions expérimentales ne seraient probablement pas les mêmes que celles de ces auteurs.

Les données obtenues à partir de ces accessions donnent une idée de la performance des accessions de niébé cultivées en Côte d’Ivoire. De 
bonnes lignées parentales pourront être obtenues en vue d'avoir des descendants à graines de grandes tailles et à haut rendement. La différence de poids de 100 graines a été observée avec respectivement 1,93 g et 18,06 g pour N15ZBoNg et N2BoBg au niveau des accessions de niébé de notre étude, cela a été déjà évoquée dans une étude similaire faite par Doumbia et al., (2013) sur des accessions de niébé du Ghana. Les résultats montrent que l'accumulation des réserves dans les graines dépend du type de génotype mais également des facteurs climatiques ( Khan, 2010). Le poids sec de 100 graines est plus élevé avec les accessions N2BoBg, N14BBoBg, et N11BBoBp, avec respectivement 18,06 g $17,18 \mathrm{~g}$, et 16,92g comparativement aux autres accessions. Ces résultats confirment ainsi l'hypothèse d'une différence dans l'efficience de la mobilisation des assimilas et partant de la capacité des accessions à assurer le remplissage des graines. La capacité à remplir les graines serait plus importante chez les accessions de N18ZR, N8BRcp, et N2BoBg qui ont ainsi exprimés les poids de graines sèches les plus élevés avec successivement 20,20 g, 19,79 g et 19,59 g. Toutefois, ces résultats ont été faibles et s'expliquerait probablement par la pauvreté du sol de culture, les conditions environnementales défavorables. Enfin, les faibles rendements observés chez la plupart des variétés pourraient s'expliquer en partie par les agressions de toutes sortes subies par les plants pendant les phases végétatives et reproductives. Ceci confirme les résultats de certains auteurs selon lesquels, dans son environnement, la plante de niébé se trouve confrontée à de nombreuses contraintes comme, les maladies, le climat (Craufurd, 2013), les ravageurs (Maïga et Issa, 1988) et même les phanérogames parasites. Le nombre de graines par plante le plus élevé a été obtenu avec la variété N21DR de niébé avec 410 graines par plante au cours de l'étude, est supérieur à celui obtenu par Djirabaye et al., (2015). Ces chercheurs, ont obtenus 20 graines par plante sur la même variété au cours d'un essai implanté dans une station de recherche localisée dans la zone soudanienne d’isohyète $1200 \mathrm{~mm}$. Un nombre réduit de graines par plante a été observé chez l'accession N13KBoNm avec 47 graines par plante. La variabilité observée dans le nombre de graines par plante traduirait une différence entre les accessions dans l'efficience de la mobilisation des assimilas vers les structures de réserve. Les coefficients de variation élevés observés pour un nombre significatif de caractères indiquent la présence d'une forte hétérogénéité au sein des accessions collectées. Les résultats pourraient être aussi en partie le reflet de la sensibilité du niébé aux variations de la photopériode (Andargie et al., 2013). En effet, de nombreux travaux ont montré que la longueur du jour entraîne des effets variables sur le développement végétatif et physiologique du niébé (Gonné et al., 2013). Les coefficients de variation élevés observés pour un nombre significatif de 
caractères indiquent la présence d’une forte hétérogénéité au sein des variétés collectées. Les accessions à cycle court qui présentent de bon rendement comme N21DR, N18ZR, N10BBrp. La précocité des variétés de niébé est une caractéristique agronomique importante qui pourrait contribuer à faire face aux phénomènes des changements climatiques. Ces accessions constituent de très bons candidats pour les programmes de sélection variétale au niveau du niébé dans le haut Sassandra.

\section{Caractérisation biochimique}

La caractérisation physicochimique des échantillons de niébé a révélé que les graines possèdent une faible humidité (7,61 \pm 0,77\%). Ceci représente un véritable atout pour la conservation de ces graines sur une période relativement longue. En effet, une teneur en eau maximale de $9 \%$ est idéale pour la conservation des graines séchées (Hayma, 2004). Famata et

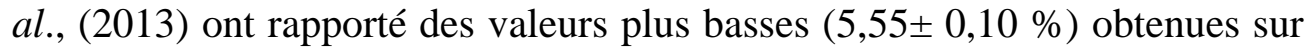
un cultivar nigérian (Kananado) de niébé. En outre, les graines de niébé contiennent des cendres dont la valeur est plus élevée que celle de 2,6 0,05 \% obtenue chez une variété améliorée (cultivar BRS-Milênio) au Brésil (Frota, 2008). La teneur en matières grasses (3,19 \%) est relativement faible comme pour la plupart des légumineuses. Ces résultats confirment ceux de Khalid et al. (2010) qui ont enregistré un taux de lipides de l'ordre de $2,1 \pm 0,1 \%$ dans une farine entière de niébé au Soudan. La richesse des échantillons analysés, en protéines (21,22 \pm 0,69\%) et en glucides (63,82 \pm $1,12 \%)$ confirme bien le fait que le niébé est une légumineuse. Des teneurs en protéines plus élevées ont été signalées avec des valeurs comprises entre 25,79 et 29,25 \% (Chinma, 2008). Par ailleurs, la teneur élevée en glucides ainsi qu'en protéines indique clairement que la graine de niébé est un aliment énergétique. Ainsi, la valeur énergétique de l’ordre de 368,92 \pm 4,71 $\mathrm{kcal} / 100 \mathrm{~g}$ en est un indicateur. De plus, la consommation d'un kilogramme de graines permet de couvrir les besoins énergétiques journaliers d'un homme adulte qui s’élèvent à $3050 \mathrm{kcal}$. La valeur énergétique enregistrée dans cette étude est plus élevée que celle obtenue par Frota, (2008) qui est de l'ordre de 323,4 kcal/100 g. Par ailleurs, des valeurs comprises entre 337 et 361 kcal/100 g de graines de niébé ont été rapportées (Chinma, 2008). En outre, au niveau des échantillons de graines, la teneur en matières grasses varie significativement d'un échantillon à l'autre (Feinberg, 1996).

L’analyse de variance a indiqué par ailleurs, que chacun des paramètres physico-chimiques des graines variait significativement d'un cultivar à l'autre. Ces paramètres permettent donc de faire la différence entre les cultivars. Il s'agit de l'humidité, des cendres, des matières grasses, des glucides, des protéines et de la valeur énergétique des graines. Les différences de teneurs enregistrées au niveau des paramètres 
physicochimiques des graines issues de différents cultivars peuvent être dues aux conditions environnementales, aux pratiques culturales et au facteur génétique (Chinma, 2008; Hamid et al., 2015). De ce fait, les graines du cultivar N19ZBoBp ont enregistré la teneur en protéines la plus élevée

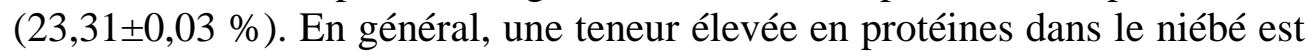
souhaitable pour une meilleure nutrition (Lambotl, 2002). Par conséquent, la teneur en protéines plus élevée observée chez le cultivar N19ZBoBp suggère qu'il pourrait être une importante source de protéines pour les populations. Agbogidi (2010) a affirmé que, le niébé est la source principale de protéines pour les populations pauvres. Cette haute teneur est par ailleurs, une indication que son utilisation en alimentation humaine pourrait aider à réduire l'incidence ou la survenue de maladies nutritionnelles tel que le kwashiorkor (Appiah et al., 2011). En outre, cette forte teneur en protéines fait que le niébé est considéré comme un excellent supplément des céréales en Afrique sahélienne (Balla et Baragé, 2006). Les cultivars N19ZBoBp principalement peuvent être recommandés à cette fin. Le cultivar N19ZBoBp possède des petites graines de couleur blanche avec un œil blanc. Hamid et al. (2015) étudiant les caractéristiques de deux variétés de niébé, ont rapporté que les graines du cultivar noir étaient plus riches en protéines $(23,23 \pm 0,44$ $\%)$ que celles du cultivar rouge (19,6 $\pm 0,04 \%)$. Dans cette étude, le cultivar rouge (N3KR) a également enregistré la valeur la plus faible $(20,60 \pm$ $0,02 \%)$.

\section{Conclusion}

La variabilité morphologique de seize accessions de niébé a été analysée par l'évaluation des paramètres à différents stades (croissance, reproduction et récolte). L'ensemble des résultats témoigne de l'existence d'une diversité importante au sein de la collection de niébé de l'Université Jean Lorougnon Guédé de Daloa. Cette importante variabilité phénotypique observée pourrait résulter de l'expression d'une forte hétérogénéité génotypique mais aussi de l'influence des facteurs environnementaux. Les cultivars N21DR, N18ZR et N10BBrp peut être recommandée aux populations pour leur production élevée en graine. Il ressort de cette étude que les graines sont des aliments énergétiques, riches en protéines et en glucides. Par contre, elles sont pauvres en cendres, en matières grasses et ont une faible humidité pouvant faciliter leur conservation sur une période relativement longue. Le cultivar N19ZBoBp ayant enregistré la teneur en protéine la plus élevée peut être utilisé en alimentation pour combler les déficits en apport protéique des populations 


\section{References:}

Agbogidi O. M.; 2010. "Response of six cultivars of cowpea (Vigna unguiculata (L.) Walp.) to spent engine oil," African Journal of Food Science and Technology, 1, (6), pp. 139-142,

Afoakwa E. O., Sefa-Dedeh S., and Sakyi-Dawson E. 2004. Effects of cowpea fortification, dehydration method and storage time on some quality characteristics of maize-based traditional weaning foods, AJFAND, Vol. 4, (1), pp. 1-15,

Andargie M, Pasquet R.S., Muluvi G.M., Timko M.P., 2013. Quantitative trait loci analysis of flowering time related traits identified in recombinant inbred lines of cowpea (Vigna unguiculata); Genome, 56: 289- 294.

Appiah F.; Asibuo J. Y., and. Kumah P, 2011. Physicochemical and functional properties of bean flours of three cowpea (Vigna unguiculata $\mathrm{L}$. Walp) varieties in Ghana, African Journal of Food Science, Vol. 5, (2), pp. $100-104$,

Balla A, et. Baragé M, 2006. Influence de la variété, du temps de stockage et du taux de natron sur la cuisson des graines de niébé, Tropicultura, 24, 1, pp. 39-44,

Chinma C. E., Alemede I. C., and. Emelife I. G., 2008. Physicochemical and Functional Properties of Some Nigerian Cowpea Varieties," Pakistan Journal of Nutrition, 7, (1), pp. 186-190,

Coulibaly, O., and Lowenberg-Deboer, J., 2002. The economics of cowpea in west Africa, In: C. A. Fatokun, S. A. Tarawali, B. B. Singh, P. M. Kormawa, and M. Tamo (Eds), Challenges and Opportinuties for enhancing sustainable cowpea production, Ibadan: IITA, pp. 354- 366, Craufurd P.Q.M., Ellis R.H., 2013. Summerfied and Menin L. Development in Cowpea Vigna unguiculata. In The influence of temperature on seed germination and seedling emergence. Experimental Agriculture, 32: 5-12.

Diallo S. K., Soro D., Koné K. Y., Assidjo N. E. Yao K. B., and D. Gnakri, 2015. Fortification et substitution de la farine de blé par la farine de Voandzou (Vigna subterranea L. verdc) dans la production des produits de boulangerie, International Journal of Innovation and Scientific Research, Vol. 18, no. 2, pp. 434-443,

Djirabaye N., Amos N.D., et Le Diambo B., 2015. Etude de la variabilité agromorphologique de quarante-cinq cultivars locaux de niébé (Vigna unguiculata, (L.)Walp.) de la zone soudanienne du Tchad, Afrique Science, 11(3), pp138 - 151

Doumbia I.Z., Akromah R., Asibuo J.Y., 2013. Comparative study of cowpea germplasms diversity from Ghana and Mali using morphological characteristics. Journal of. Plant Breeding. Genetic , 01(03), pp139-147. 
Famata A. S., Modu S. Mida H. M., Hajjagana L., Shettima A. Y., and. Hadiza A, 2013. Chemical composition and mineral element content of two cowpea (Vigna unguiculata L. walp) varieties as food supplement, International Research Journal of Biochemistry and Bioinformatics, Vol. 3, (4), pp. 93-96,

Feinberg M., 1996. La validation des méthodes d'analyse : Approche Chimiométrique de l'assurance qualité au laboratoire, Ed. Masson,

Frota K. M. G., Soares R. A. M., and. Arêas J. A.G., 2008. Chemical composition of cowpea (Vigna unguiculata L. Walp), BRS-Milênio cultivar, Ciênc. Tecnologic. Aliment., Campinas, 28, (2), pp. 470-476,

Gonné S, Wirnkar L.V., Laminou A., 2013. Characterization of Some Traditional Cowpea Varieties Grown by Farmers in the SoudanoSahelian Zone of Cameroon. International Journal of Agriculture and Forestry, 3(4): 170-177.

Hamid S., Muzzafar S.; Wani I. A., and. Masoodi F. A., 2015. Physicochemical and functional properties of two cowpea cultivars grown in temperate Indian climate,” Cogent Food \& Agriculture, 1: 1099418, Hayma J., 2004. Le stockage des produits agricoles. Agrodok 31, 4 Edition. Digigrafi, Wageningen, The Netherlands,

Houinsou R. L. F. Adjou E., Sahoussi D. E., Sohounhloué D. C. K., and. Soumanou M. M, 2014. Caractéristiques biochimique et sensorielle du niébé (Vigna unguiculata) conservé au moyen des huiles essentielles extraites de plantes de la famille des Myrtaceae, International Journal of Innovation and Applied Studies, Vol. 9, no. 1, pp. 428-437,

Khalid I. I., Elhardallou B., and. Elkhalifa E. A., 2012. Composition and functional properties of cowpea (Vigna unguiculata, (L.) Walp) flour and protein isolates, American Journal of food technology, 7, (3), pp. 113-122,

Khan A, Bari A., Khan S., Shan N.H, Zada I., 2010. Performance of cowpea genotypes at higher altitude of NWFP. Pakistan. Journal of.

Botanic., 42(4): 2291-2296.

Lambot C.; 2002. Industrial potential of cowpea. In: C. A. Fatokun, S. A. Tarawali, B. B. Singh, P. M. Kormawa, and M. Tamo (Eds), Challenges and Opportinuties for enhancing sustainable cowpea production, Ibadan: IITA, pp: 367-375,

Maïga S., et Issa H., 1988. Les principaux insectes nuisibles aux cultures pluviales. Dans ', Manuel de l'expérimentation en plein champs'inran,Niamey, Niger, 66-92.

N’gbesso F. P. M., Fondio L., Dibi K. B. E, Djidji A. H., et Kouamé N. C., 2013. Étude des composantes du rendement de six variétés améliorées de niébé [Vigna unguiculata (L.) Walp], Journal of Applied Biosciences, 63, pp. 4754 - 4762, 
Ohimain $\mu$, 2014. The Prospects and Challenges of Cassava Inclusion in Wheat Bread Policy in Nigeria, International Journal of Science, Technology and Society, 2, (1), pp. 6-17,

Pasquet, S. R., et Baudoin, J. P., Le niébé, In : A. 1997. Charrier, M. Jacquot, S. Hamon, and D. Nicolas (Eds.), L'amélioration des plantes tropicales, Montpellier : CIRAD, ORSTOM, pp. 483-505,

Touko, A. B., Egue, K., Goto, C. E., Sedzro, K., Tougnon, K., Amouzou, A., 2007. Promotion du manioc par la diversification de ses formes d'utilisation, In: G. Amani, C. Nindjin, B. N’zué, A. Tschannen, D. Aka (Eds), Atelier International sur les Potentialités à la Transformation du Manioc en Afrique de l'Ouest, Abidjan : FIDAfrique, pp. 120-122,

Tableau 1: Méthodes de mesures et paramètres étudiés sur cinq plantes par accession.

\begin{tabular}{|c|c|}
\hline Paramètres mesurés & Méthodes de mesures \\
\hline Nombre de gousses :NGs & $\begin{array}{c}\text { Le nombre de gousses a été déterminé en } \\
\text { comptant l'ensemble des gousses des plantes. } \\
\text { Les gousses sèches des plantes ont été pesées } \\
\text { à l'aide d'une balance électronique de } \\
\text { précision. }\end{array}$ \\
Poids des gousses sèches vides : pds (g) : & $\begin{array}{c}\text { Les gousses sèches vides des plantes ont été } \\
\text { pesées à l'aide d'une balance électronique de } \\
\text { précision. }\end{array}$ \\
Nombre de graines : NbGr $(\mathrm{g}):$ PGoS & $\begin{array}{c}\text { Le nombre de graines a étévalué en } \\
\text { comptant l'ensemble des graines après } \\
\text { décorticage de gousses. }\end{array}$ \\
Poids de 100 graines (g) :Pgr100 & $\begin{array}{c}\text { le comptage de 100 graines a été effectué } \\
\text { manuellement et le poids à l'aide d'une } \\
\text { balance électronique. }\end{array}$ \\
Poids des graines sèches $(\mathrm{g}):$ PGrs & $\begin{array}{c}\text { Les graines sèches des plantes ont été pesées } \\
\text { à l'aide d'une balance électronique de } \\
\text { précision. }\end{array}$ \\
Indice de récolte : InR & $\begin{array}{c}\text { Rapport entre le poids des graines sèches des } \\
\text { plantes sur la biomasse sèche totale. } \\
\text { Le rapport du Poids des graines sèches des } \\
\text { plantes sur le poids des gousses des mêmes } \\
\text { plantes. }\end{array}$ \\
\hline
\end{tabular}


Tableau 2. Moyennes ( \pm écart-type) des caractères agronomiques mesurés sur les différentes accessions

\begin{tabular}{|c|c|c|c|c|c|c|c|c|}
\hline accessions & NGs & NbGr & PGos(g) & Pgr(100gr) & Pds coq(g) & Pgr & InR & TR \\
\hline N3KR & $19.20 \pm 6.76 \mathrm{c}$ & $241.60 \pm 89.48 \mathrm{de}$ & $25.76 \pm 5.11 \mathrm{abc}$ & $10.36 \pm 1.10 \mathrm{de}$ & $6.06 \pm 2.55$ abc & $15.43 \pm 2.72 \mathrm{de}$ & $0.35 \pm 0.11 \mathrm{~cd}$ & $0.60 \pm 0.78 \mathrm{ij}$ \\
\hline N5BBr & $17.20 \pm 4.14 \mathrm{bc}$ & $231.60 \pm 77.37 \mathrm{~d}$ & $26.65 \pm 3.78 \mathrm{~cd}$ & $11.04 \pm 1.02$ de & $9.09 \pm 1.73$ bcdef & $12.77 \pm 2.37 \mathrm{~cd}$ & $0.20 \pm 0.04 \mathrm{abc}$ & $0.47 \pm 0.03$ defgh \\
\hline N6BR & $18.40 \pm 3.28 b c$ & $240.20 \pm 61.50 \mathrm{de}$ & $27.09 \pm 1.30 \mathrm{~cd}$ & $9.50 \pm 0.26$ cde & $7.02 \pm 1.25$ abcde & $15.93 \pm 1.00 \mathrm{de}$ & $0.28 \pm 0.05 \mathrm{bcd}$ & $0.58 \pm 0.03 \mathrm{hij}$ \\
\hline N7BRc & $19.80 \pm 5.97 \mathrm{c}$ & $263.20 \pm 78.13 \mathrm{def}$ & $35.62 \pm 5.35$ def & $11.79 \pm 3.34 \mathrm{e}$ & $12.53 \pm 3.49 \mathrm{eg}$ & $15.41 \pm 3.43$ de & $0.18 \pm 0.05 a b c$ & $0.43 \pm 0.12$ cdefg \\
\hline N8BRcp & $27.60 \pm 8.04 \mathrm{de}$ & $367 \pm 133.27 \mathrm{fg}$ & $36.23 \pm 15.82 \mathrm{def}$ & $9.04 \pm 2.11$ cde & $10.79 \pm 5.91 \mathrm{ef}$ & $19.79 \pm 7.80 \mathrm{e}$ & $0.27 \pm 0.11 \mathrm{bcd}$ & $0.54 \pm 0.05$ ghij \\
\hline N9BN & $16.80 \pm 3.34$ bc & $250.80 \pm 80.62 \mathrm{de}$ & $29.83 \pm 6.43 \mathrm{de}$ & $11.41 \pm 0.39 \mathrm{de}$ & $8.32 \pm 1.72$ bcde & $16.96 \pm 3.79 \mathrm{de}$ & $0.28 \pm 0.05 \mathrm{bcd}$ & $0.56 \pm 0.03 \mathrm{hij}$ \\
\hline N10BBrp & $23.20 \pm 7.69 \mathrm{~cd}$ & $355.40 \pm 165.71 \mathrm{efg}$ & $35.10 \pm 8.60 \mathrm{def}$ & $10.80 \pm 3.18 \mathrm{de}$ & $0.37 \pm 3.69$ def & $20.15 \pm 5.54 \mathrm{e}$ & $0.28 \pm 0.05 \mathrm{bcd}$ & $0.52 \pm 0.12$ fghij \\
\hline N15ZBoNg & $8 \pm 0.70 \mathrm{a}$ & $65.2 \pm 21.60 \mathrm{ab}$ & $14.72 \pm 2.564 \mathrm{ab}$ & $1.93 \pm 1.00 \mathrm{a}$ & $7 . .47 \pm 1.84$ bcde & $1.46 \pm 0.88 \mathrm{a}$ & $0.01 \pm 0.00 \mathrm{a}$ & $0.09 \pm 0.06 \mathrm{a}$ \\
\hline N18ZR & $27.40 \pm 10.31 \mathrm{de}$ & $381.80 \pm 186.62 \mathrm{~g}$ & $34.34 \pm 13.70 \mathrm{def}$ & $9.64 \pm 1.04$ cde & $9.76 \pm 4.94$ cdef & $20.20 \pm 5.69 \mathrm{e}$ & $0.29 \pm 0.11 \mathrm{bcd}$ & $0.61 \pm 0.13 \mathrm{j}$ \\
\hline N21DR & $30.60 \pm 7.33 \mathrm{e}$ & $409.80 \pm 104.05 \mathrm{~g}$ & $41.23 \pm 13.75 \mathrm{f}$ & $10.81 \pm 2.10 \mathrm{de}$ & $15.21 \pm 5.32 \mathrm{~g}$ & $18.49 \pm 3.19 \mathrm{e}$ & $0.21 \pm 0.04 \mathrm{abc}$ & $0.48 \pm 0.21$ efghi \\
\hline N2BoBg & $18.80 \pm 1.64 \mathrm{c}$ & $175.40 \pm 35.02 \mathrm{bcd}$ & $36.04 \pm 8.93 \mathrm{def}$ & $18.06 \pm 1.34 \mathrm{f}$ & $8.27 \pm 3.49$ bcde & $19.59 \pm 1.96 \mathrm{e}$ & $0.36 \pm 0.04 \mathrm{~cd}$ & $0.55 \pm 0.11 \mathrm{hij}$ \\
\hline N4KBNp & $11.40 \pm 1.34 \mathrm{ab}$ & $107.00 \pm 17.79 a b c$ & $25.89 \pm 3.11 \mathrm{~cd}$ & $8.51 \pm 1.06 \mathrm{~cd}$ & $6.53 \pm 1.67 \mathrm{abcd}$ & $8.41 \pm 1.04 \mathrm{bc}$ & $0.50 \pm 1.04 \mathrm{~d}$ & $0.32 \pm 0.05 \mathrm{bc}$ \\
\hline N11BBoBp & $22.00 \pm 7.84 \mathrm{~cd}$ & $190.20 \pm 85.11 \mathrm{~cd}$ & $40.20 \pm 14.49$ ef & $16.92 \pm 4.29 \mathrm{f}$ & $9.53 \pm 2.96 \mathrm{cdef}$ & $17.08 \pm 5.55$ de & $0.23 \pm 0.08 \mathrm{abc}$ & $0.42 \pm 0.04$ bcdef \\
\hline N13KBoNm & $7.80 \pm 3.11 \mathrm{a}$ & $47.40 \pm 22.68 \mathrm{a}$ & $18.68 \pm 7.03 \mathrm{abc}$ & $6.74 \pm 2.07 \mathrm{bc}$ & $5.17 \pm 2.10 \mathrm{ab}$ & $6.71 \pm 2.05 b$ & $0.11 \pm 0.05 \mathrm{ab}$ & $0.36 \pm 0.06 \mathrm{bcd}$ \\
\hline N14BBoBg & $20.00 \pm 2.12 c$ & $173.60 \pm 19.44 \mathrm{bcd}$ & $41.38 \pm 2.68 \mathrm{f}$ & $17.18 \pm 4.21 \mathrm{f}$ & $10.19 \pm 0.54 \mathrm{def}$ & $16.67 \pm 3.63 \mathrm{de}$ & $0.23 \pm 0.09 \mathrm{abc}$ & $0.39 \pm 0.06$ bcde \\
\hline N19ZBoBp & $9.20 \pm 5.06 \mathrm{a}$ & $77.80 \pm 53.70 a b c$ & $12.16 \pm 7.49 a$ & $4.75 \pm 2.46 \mathrm{ab}$ & $3.39 \pm 2.16 \mathrm{a}$ & $3.91 \pm 2.70 \mathrm{ab}$ & $0.08 \pm 0.05 \mathrm{ab}$ & $0.31 \pm 0.02 \mathrm{~b}$ \\
\hline
\end{tabular}

NB Les valeurs portant les mêmes lettres sur la colonne sont statistiquement égales. Les abréviations sont indiquées dans le tableau 1 
Tableau 3 Moyennes ( \pm écart-type) des caractères biochimiques mesurés sur les différentes accessions

\begin{tabular}{|c|c|c|c|c|c|c|}
\hline Variétés & $\begin{array}{c}\text { Humidité } \\
(\%)\end{array}$ & $\begin{array}{l}\text { Cendres } \\
\text { (\%) }\end{array}$ & $\begin{array}{c}\text { Matière } \\
\text { grasse (\%) }\end{array}$ & $\begin{array}{l}\text { Glucides } \\
\text { (\%) }\end{array}$ & $\begin{array}{l}\text { Protéines } \\
\text { (\%) }\end{array}$ & $\begin{array}{c}\text { Valeur } \\
\text { énergétique } \\
\text { (kcal/100 g) }\end{array}$ \\
\hline N19ZBoBp & $7 \underset{\mathrm{a}}{7,29 \pm 0,05}$ & $\underset{\mathrm{a}}{4,18 \pm 0,03}$ & $2, \frac{a}{a}+0,12$ & $\underset{\mathrm{a}}{62,88 \pm 0,15}$ & $23,31 \pm 0,03^{\mathrm{a}}$ & $365,72 \pm 0,79^{a}$ \\
\hline N2KBoBg & $7,95 \underset{b}{ \pm} 0,02$ & $3,94 \underset{b}{ \pm} 0,02$ & $4,01 \pm 0,16$ & $\underset{\mathrm{a}}{62,90 \pm 0,13}$ & $21,18 \pm 0,03^{b}$ & $372,46 \pm 0,80^{b}$ \\
\hline N4KBNp & $6,31 \pm \underset{c}{ \pm 0,02}$ & $\underset{\mathrm{c}, \mathrm{b}}{3,94 \pm 0,01}$ & $\begin{array}{c}3,37 \pm 0,15 \\
\mathrm{C}\end{array}$ & $\underset{\mathrm{b}}{65} \underset{\mathrm{f}}{72 \pm 0,13}$ & $20,64 \pm 0,08^{\mathrm{c}}$ & $375,84 \pm 0,78^{\mathrm{C}}$ \\
\hline $\mathrm{N} 5 \mathrm{BBr}$ & $7,17 \pm 0,01$ & $4,30 \pm 0,01$ & $\begin{array}{c}3,65 \pm 0,06 \\
\text { d, b, c }\end{array}$ & $\underset{\mathrm{a}}{63,28 \pm 0,56}$ & $21,25 \pm 0,06^{\mathrm{d}}$ & $370,99 \pm 2,02^{\text {d, b }}$ \\
\hline N8BRcp & $7,4 \underset{\mathrm{e}}{ \pm 0,02}$ & $\underset{\mathrm{d}, \mathrm{b}, \mathrm{j}}{4,02 \pm 0,02}$ & $\begin{array}{c}3,54 \pm 0,21 \\
\text { e, c }\end{array}$ & $\underset{\mathrm{c}}{63,73 \pm 0,24}$ & $21,31 \pm \underset{, b}{0} 0,04^{e}$ & $372,07 \pm 0,98^{e, b}$ \\
\hline N9BN & $7,68 \pm 0,03^{f}$ & $\begin{array}{c}3,88 \pm 0,01 \\
\text { e,b }\end{array}$ & $\begin{array}{c}3,18 \pm 0,09^{f} \\
, c\end{array}$ & $64,4 \underset{d}{6} 0,15$ & $20,8 \underbrace{ \pm 0}_{c} 0,07^{f}$ & $369,63 \pm 0,51^{\mathrm{f}, \mathrm{d}, \mathrm{e}}$ \\
\hline$\underset{\mathrm{m}}{\mathrm{N} 13 \mathrm{KBoN}}$ & $8 \underset{\mathrm{g}}{8,52 \pm 0,03}$ & $\underset{\mathrm{a}}{4,23 \pm 0,01}$ & $2, \underset{\mathrm{a}}{2,65} \mathbf{0}, 15$ & $\underset{\mathrm{e}}{63,53 \pm 0,07}$ & $21,07 \pm 0,05^{g}$ & $362,25 \pm 0,86^{\mathrm{g}}$ \\
\hline N1KBN & $\underset{\mathrm{h}, \mathrm{b}}{7,96 \pm 0,01}$ & $\underset{, b}{3,88 \pm 0,06^{f}}$ & $1,95 \underset{a}{a} 0,08$ & $64,10 \pm 0,07^{f}$ & $22,11 \pm 0,07^{\mathrm{h}}$ & $362,39 \pm 0,56^{\text {h, g }}$ \\
\hline N3KR & $7,03 \pm 0,01^{\mathrm{i}}$ & $\underset{\mathrm{a}, \mathrm{j}}{ \pm, 17} \underset{0,02}{ }$ & $\underset{\mathrm{g}, \mathrm{c}}{3,61 \pm 0,09}$ & $\underset{\mathrm{g}}{64,54 \pm 0,09}$ & $20, \underset{c}{63 \pm 0,02^{i}}$ & $373,23 \pm 0,45^{\mathrm{i}, \mathrm{b}, \mathrm{e}}$ \\
\hline N7BRc & $\underset{, f}{7,64 \pm 0,01^{j}}$ & $\underset{\mathrm{g}, \mathrm{b}, \mathrm{j}}{4,00 \pm 0,01}$ & $\underset{\mathrm{h}, \mathrm{c}}{3,28 \pm 0,21}$ & $\underset{\mathrm{h}}{64,28 \pm 0,17}$ & $20, \underset{c}{78} \pm 0,08^{j}$ & $\underset{\mathrm{e}, \mathrm{f}}{369,86 \pm 1,02^{\mathrm{j}, \mathrm{d}}}$ \\
\hline N10BBrp & $8,40 \pm \frac{ \pm}{\mathrm{k}} 0,01$ & $\underset{\mathrm{a}, \mathrm{j}}{4,16 \pm 0,01}$ & $\begin{array}{l}3,27 \pm 0,08^{i} \\
, c\end{array}$ & $\underset{\mathrm{a}}{63,15 \pm 0,05}$ & $\underset{, \mathrm{b}, \mathrm{f}, \mathrm{j}}{21,00 \pm 0,14^{\mathrm{k}}}$ & $366,11 \pm 0,42^{\mathrm{a}}$ \\
\hline$\underset{\mathrm{m}}{\mathrm{N} 12 \mathrm{KBoB}}$ & $9,33 \pm 0,01^{\mathrm{l}}$ & $4,90 \pm 0,05$ & $\underset{, c}{3,52 \pm 0,06^{\mathrm{j}}}$ & $61,44 \pm 0,14^{\mathrm{i}}$ & $\underset{c, f, j}{20,79 \pm 0,15^{l}}$ & $360,68 \pm \frac{ \pm}{h} 0,21^{k, g}$ \\
\hline N15ZBoNg & $\underset{\mathrm{a}}{7,32 \pm 0,03}$ & $\underset{, b}{3,83 \pm 0,15^{\mathrm{i}}}$ & $\underset{\mathrm{k}, \mathrm{b}}{3,79 \pm 0,04}$ & $63,74 \pm 0,11^{\mathrm{j}}$ & $\underset{\mathrm{m}, \mathrm{b}}{21,30 \pm 0,07}$ & 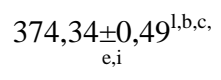 \\
\hline N17ZBoNp & $\underset{\mathrm{m}}{6,52 \pm 0,03}$ & $4 \underset{\mathrm{a}}{4,23 \pm 0,02}$ & $2,49 \pm \underset{a}{0} 0,11$ & $\underset{\mathrm{k}, \mathrm{b}}{65,81 \pm 0,02}$ & $\underset{, f, \mathrm{~g}, \mathrm{j}}{20,93 \pm 0,09^{\mathrm{n}}}$ & 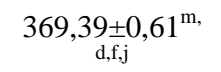 \\
\hline
\end{tabular}

Les teneurs avec des lettres identiques dans la même colonne sont statistiquement identiques (risque de $5 \%$ ). 